\title{
Rotor Flux Estimation of Induction Motors Using Sliding-Mode Observer
}

\author{
Yong Feng ${ }^{1, \mathrm{a}}$, Minghao Zhou ${ }^{1, \mathrm{~b}}$ and Fengling $\mathrm{Han}^{2, \mathrm{c}}$ \\ ${ }^{1}$ Department of Electrical Engineering, Harbin Institute of Technology, China \\ ${ }^{2}$ School of Science, RMIT University, Australia \\ ayfeng@hit.edu.cn, ${ }^{b}$ zhouminghao@hit.edu.cn, ${ }^{c}$ fengling.han@rmit.edu.au
}

Keywords: Rotor flux observers; sliding-mode systems; induction motors.

Abstract. A high-order nonsingular terminal sliding-mode observer is presented for rotor flux of induction motors based on field oriented control systems in this paper. A terminal sliding-mode manifold is chosen for the observer and the nonsingular control laws are utilized to stabilize the observer. The high-order sliding-mode control method is applied to guarantee the strong robustness of the induction motor system and to produce a smooth control signal of the observer. The soften signal is directly used for the rotor flux estimation. The effect of the equivalent low-pass filter in the proposed observer can be optionally regulated. The chattering phenomenon can be attenuated effectively. The simulation results validate the proposed method.

\section{Introduction}

Induction motors (IMs) have been extensively used in many practical applications in consideration of the simple construction, lower repair and maintenance costs, high reliability and relatively low manufacturing cost, [1]. The scalar control, the field oriented control (FOC) and the direct torque control (DTC) are three main methods for the control of IMs, and especially both the latter two methods can provide high-performance control for the motor servo systems.

The DTC method for IMs applies the bang-bang control to regulate the stator flux and the torque independently. The weakness of this method is that the torque has ripple. Nevertheless, in FOC-based IM motor systems the torque and the flux of the motor can be controlled continuously. The decoupled torque and the rotor flux of an IM can be controlled separately like DC motors and a high steady-state and dynamic performances of IMs can be achieved [2].

The FOC of an IM needs the information of the rotor flux. This information can be obtained by either direct measurements or indirect estimation methods. The special sensors inhibits the practical applications of the direct measurements while at present the indirect estimation methods have been utilized for obtaining the rotor flux [3], i.e., the rotor flux can be estimated by using the measurements of the stator voltages and currents. A lot of estimation methods have been proposed [4,5,6], etc.

The robustness to disturbances and low sensitivity to the system parameter variations are the attractive advantages of sliding-mode control [7] and [8]. Sliding-mode observer can be applied for the estimation of the rotor flux of IMs. However the chattering phenomena existing in conventional sliding-mode control limits its applications [9] and [10].

This paper presents an estimation method for rotor flux of IMs based on high-order nonsingular terminal sliding-mode control. The rotor flux of the IM can be estimated using the smooth control signal generated by the observer. The simulation results validate the proposed method.

\section{Dynamic Model of Induction Motors}

The dynamic model of an IM can be described in two-dimensional static frame $(\alpha-\beta)$ as follows [2]:

$$
\left\{\begin{array}{l}
\frac{d i_{\alpha s}}{d t}=K\left(u_{\alpha s}-R_{s} i_{\alpha s}\right)+K\left(\eta \phi_{\alpha s}+\omega_{r} \phi_{\beta s}\right)-\frac{\eta}{\sigma} i_{\alpha s}-\omega_{r} i_{\beta s} \\
\frac{d i_{\beta s}}{d t}=K\left(u_{\beta s}-R_{s} i_{\beta s}\right)+K\left(-\omega_{r} \phi_{\alpha s}+\eta \phi_{\beta s}\right)+\omega_{r} i_{\alpha s}-\frac{\eta}{\sigma} i_{\beta s}
\end{array}\right.
$$


where $K=1 /\left(\sigma L_{s}\right), \sigma=1-L_{m}^{2} /\left(L_{s} L_{r}\right), \eta=1 / T_{r}=R_{r} / L_{r}, u_{\alpha s}$ and $u_{\beta s}$ are the stator voltages in $\alpha-\beta$ axes, $i_{\alpha s}$ and $i_{\beta s}$ the stator currents in $\alpha-\beta$ axes, $\phi_{a s}$ and $\phi_{\beta s}$ the stator fluxes in $\alpha-\beta$ axis, $\omega_{r}$ the electric angular velocity of the rotor, $R_{s}$ the stator resistor, $L_{r}, L_{s}$, and $L_{m}$ the rotor, the stator, and the mutual inductance between the stator and the rotor, $T_{r}$ the time constant of the rotor.

The relationship between the rotor and the stator fluxes of can be expressed as follows:

$$
\left\{\begin{array}{l}
\phi_{\alpha r}=\frac{L_{r}}{L_{m}}\left(\phi_{\alpha s}-\sigma L_{s} i_{\alpha s}\right) \\
\phi_{\beta r}=\frac{L_{r}}{L_{m}}\left(\phi_{\beta s}-\sigma L_{s} i_{\beta s}\right)
\end{array} .\right.
$$

where $\phi_{\alpha r}$ and $\phi_{\beta r}$ are the rotor fluxes in $\alpha-\beta$ axis.

From Eq.(1), the information of the stator fluxes in an IM are contained in both $K\left(\eta \phi_{\alpha s}+\omega_{r} \phi_{\beta s}\right)$ and $K\left(-\omega_{r} \phi_{\alpha s}+\eta \phi_{\beta s}\right)$. Considering the relationship between the rotor fluxes and the stator fluxes, it can be seen that these information including both the rotor and stator fluxes can be obtained.

\section{Sliding-Mode Observer for Rotor Flux}

The sliding-mode observer-based vector control system of an IM is shown in Fig.1. The observer is used to estimate the rotor flux of the IM. The inputs of the observer include the stator currents and voltages of the IM.

\section{A. Estimation of the Stator Currents}

An observer for the stator currents is built as follows:

$$
\left\{\begin{array}{l}
\underset{l_{\alpha s}}{\&}=K u_{\alpha s}+v_{\alpha s}-\left(K R_{s}+\frac{\eta}{\sigma}\right) \hat{i}_{\alpha s}-\omega_{r} \hat{i}_{\beta s} \\
\underset{l_{\beta s}}{\&}=K u_{\beta s}+v_{\beta s}+\omega_{r} \hat{i}_{\alpha s}-\left(K R_{s}+\frac{\eta}{\sigma}\right) \hat{i}_{\beta s}
\end{array}\right.
$$

where $\hat{i}_{\alpha s}$ and $\hat{i}_{\beta s}$ are the estimates of the stator currents $i_{\alpha s}$ and $i_{\beta s}, v_{\alpha s}$ and $v_{\beta s}$ are the control signals of the observer, which replace the terms $K\left(\eta \phi_{\alpha s}+\omega_{r} \phi_{\beta s}\right)$ and $K\left(-\omega_{r} \phi_{\alpha s}+\eta \phi_{\beta s}\right)$ in Eq.(1).

From Eqs. (1) and (3), it can be obtained the error observer as follows:

$$
i^{\alpha}=v-K P+N \bar{i}
$$

where $\overline{\boldsymbol{i}}=\left[\bar{i}_{\alpha s}, \bar{i}_{\beta s}\right]^{\mathrm{T}}, \quad \bar{i}_{\alpha s}=\hat{i}_{\alpha s}-i_{\alpha s}$ and $\bar{i}_{\beta s}=\hat{i}_{\beta s}-i_{\beta s}$ are the estimation errors of the currents in $\alpha-\beta$ axis, $\boldsymbol{P}=\left[K\left(\eta \phi_{\alpha s}+\omega_{r} \phi_{\beta s}\right), K\left(-\omega_{r} \phi_{\alpha s}+\eta \phi_{\beta s}\right)\right]^{T}, \boldsymbol{v}=\left[v_{\alpha s}, v_{\beta s}\right]^{\mathrm{T}}$, and

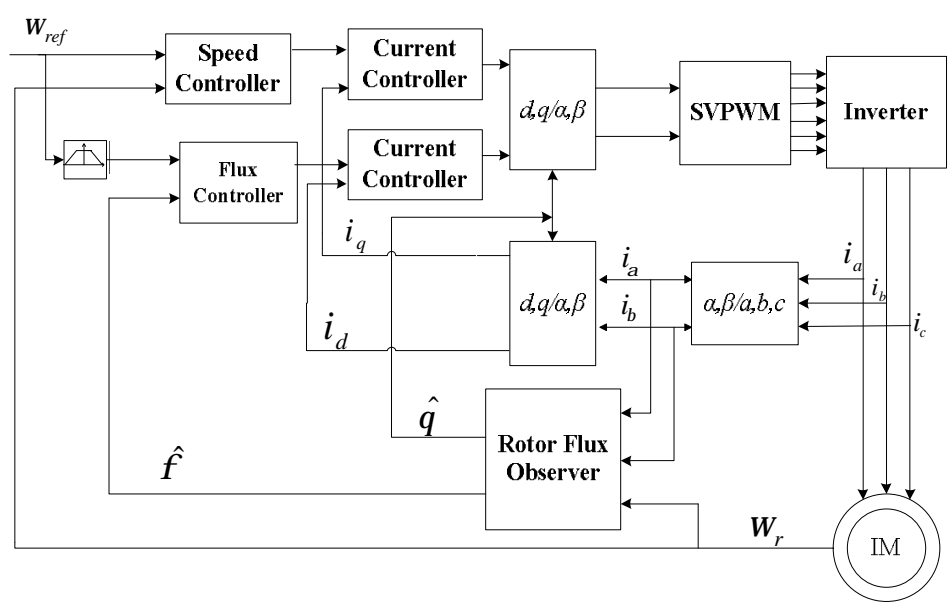

Fig. 1 IM vector control system. 


$$
N=\left[\begin{array}{ll}
-\left(\frac{\eta}{\sigma}+K R_{s}\right) & -\omega_{r} \\
\omega_{r} & -\left(\frac{\eta}{\sigma}+K R_{s}\right)
\end{array}\right] .
$$

A nonsingular terminal sliding-mode manifold is designed as:

$$
s=\bar{i}+\gamma \boldsymbol{l}^{\boldsymbol{\delta}_{\phi / q}}
$$

where $s=\left[s_{\alpha s}, s_{\beta s}\right]^{T}, \gamma=\operatorname{diag}\left(\gamma_{\alpha s}, \gamma_{\beta s}\right), \gamma_{\alpha s}>0, \gamma_{\beta s}>0 . p$ and $q$ are odds and satisfy $1<p / q<2$.

It can be assumed that system (1) and its observer (3) satisfy $\|\boldsymbol{T} \boldsymbol{v}\|<k_{1},\|K \boldsymbol{R}\|<k_{2}$, where $k_{1}, k_{2}$, and $T$ are positive constants.

The observer error $\bar{i}$ and its derivative in (4) will converge to zero in finite time, if a NTSM manifold is chosen as (5) and the control strategy is designed as follows:

$$
\begin{aligned}
& v+T v=w
\end{aligned}
$$

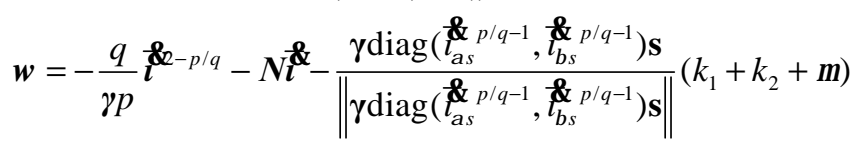

where $\mu$ is a positive constant.

\section{B. Estimation of the Rotor Fluxes}

If the ideal sliding-mode motion $s=0$ is guaranteed to be implemented using Theorem 1 , the observer error and its derivative satisfy $\overline{\boldsymbol{i}}=\hat{\boldsymbol{t}}=0$, and (4) can be expressed as:

$$
\left\{\begin{array}{l}
v_{\alpha s}=K\left(\eta \phi_{\alpha s}+\omega_{r} \phi_{\beta s}\right) \\
v_{\beta s}=K\left(-\omega_{r} \phi_{\alpha s}+\eta \phi_{\beta s}\right)
\end{array} .\right.
$$

The estimates of the stator fluxes can be obtained from Eq.(8):

$$
\left\{\begin{array}{l}
\hat{\phi}_{\alpha s}=\frac{\eta}{K\left(\eta^{2}+\omega^{2}{ }_{r}\right)} v_{\alpha s}-\frac{\omega_{r}}{K\left(\eta^{2}+\omega^{2}{ }_{r}\right)} v_{\beta s} \\
\hat{\phi}_{\beta s}=\frac{\omega_{r}}{K\left(\eta^{2}+\omega_{r}{ }_{r}\right)} v_{\alpha s}+\frac{\eta}{K\left(\eta^{2}+\omega^{2}{ }_{r}\right)} v_{\beta s}
\end{array} .\right.
$$

According to Eqs. (2), the estimates of the rotor fluxes can be obtained from Eqs.(3) and (9):

$$
\left\{\begin{array}{l}
\hat{\phi}_{\alpha r}=\frac{L_{r}}{L_{m}}\left(\hat{\phi}_{\alpha s}-\sigma L_{s} \hat{i}_{\alpha s}\right) \\
\hat{\phi}_{\beta r}=\frac{L_{r}}{L_{m}}\left(\hat{\phi}_{\beta s}-\sigma L_{s} \hat{i}_{\beta s}\right)
\end{array} .\right.
$$

Consequently both the magnitude and the position of the rotor fluxes are obtained, which can be utilized in the FOC based IM system.

\section{Simulations}

A three phase squirrel-cage IM is considered in the simulation. Assume that the parameters of the IM are: $P_{N}=1.5 \mathrm{~kW}, U_{N}=380 \mathrm{~V}, R_{r}=3.2 \Omega, R_{s}=6.1 \Omega, L_{r}=0.478 \mathrm{H}, L_{s}=0.478 \mathrm{H}, L_{m}=0.472 \mathrm{H}, J=0.033 \mathrm{~kg} \cdot \mathrm{m}^{2}$.

The design parameters of the rotor flux observer are: $p=5, q=3, \gamma=1, T=0.001, k_{1}+k_{2}=8000$.

The simulation results are shown in Figs. 2 and 3. The actual and the estimated rotor fluxes of the IM in $\alpha$ axis are shown in Fig.2. The actual and the estimated rotor fluxes of the IM are explained in Fig.3. It can be seen from the simulation results that the proposed method can estimate the rotor flux of the IM quickly and actually. 


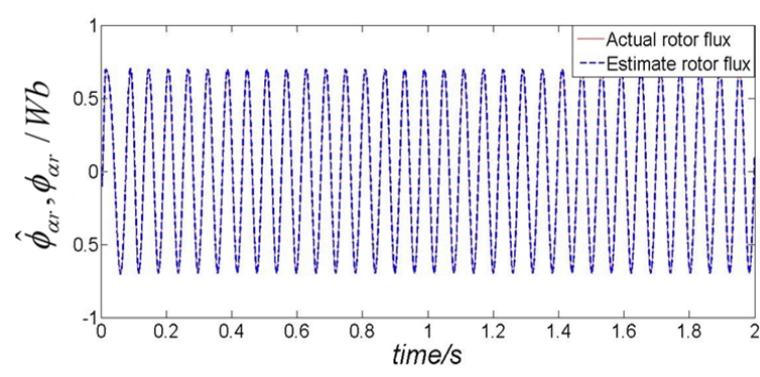

Fig. 2 Actual rotor flux and its estimation in $\alpha$ axis.

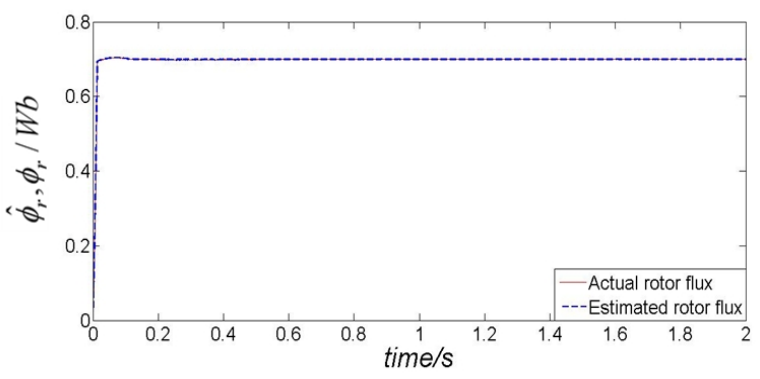

Fig. 3 Actual rotor flux and its estimation.

\section{Conclusions}

This paper has proposed a high-order sliding-mode observer based estimation method for the rotor flux of IMs. A nonsingular terminal sliding-mode surface and a control strategy of the observer are designed to make the observer track the stator currents. A smooth control signal of the observer can be generated by utilizing the second-order sliding-mode technique, and directly used for estimation of the rotor flux. The estimated rotor flux can be used for implementing the FOC of the IM systems. The simulation results have shown the correctness of the proposed method.

\section{Acknowledgements}

This work was financially supported by National Natural Science Foundation (NNSF) of China under Grant (61673132).

\section{References}

[1] Maes and J. Melkebeek, "Speed-sensorless direct torque control of induction motors using an adaptive flux observer," IEEE Trans. Ind. Appl., vol.36, no.3, pp. 778-785, 2000.

[2] S. Kwon, M.H. Shin, and D.S. Hyun, "Speed sensorless stator flux-oriented control of induction motor in the field-weakening region using Luenberger observer," IEEE Trans. Power Electron., vol.20, no.4, pp.864-869, 2005.

[3] M. S. Zaky, "Stability analysis of speed and stator resistance estimators for sensorless induction motor drives," IEEE Trans. Ind. Electron., vol.59, no.2, pp.858-70, 2012.

[4] F. Chen and M. Dunnigan, "Comparative study of a sliding-mode observer and Kalman filters for full state estimation in an induction machine," IEE Proc., Electr. Power Appl., vol.149, no.1, pp.53-64, 2002.

[5] S. Kim, T. Park, and G. Park, "Speed-sensorless vector control of an induction motor using neural network estimation," IEEE Trans. Power Electron., vol.48, no.3, pp.609-614, 2001.

[6] Y. Feng, X. Yu and F. Han, "High-order terminal sliding-mode observer for parameter estimation of a permanent-magnet synchronous motor," IEEE Trans. Ind. Electron., vol.60, no.10, pp. 4272-4280, Oct. 2013.

[7] Y. Feng, X. Yu, and Z. Man, "Non-singular terminal sliding mode control of rigid manipulators," Automatica, vol.38, no.12, pp.2159-2167, 2002.

[8] Y. Feng, X. Yu and F. Han, "High-order terminal sliding-mode observer for parameter estimation of a permanent-magnet synchronous motor," IEEE Trans. Ind. Electron., vol.60, no.10, pp. 4272-4280, Oct. 2013.

[9] Y. Feng, X. Yu, and F. Han, "On nonsingular terminal sliding-mode control of nonlinear systems," Automatica, vol.46, no.6, pp. 1715-1722, June 2013. 
[10] Y. Feng, X. Yu, F. Han, "Chattering free full-order sliding-mode control", Automatica, vol.50, no.4, pp.1310-1314, 2014. 